\title{
Prognostic importance of DNA ploidy in non-endometrioid, high-risk endometrial carcinomas
}

\author{
BENGT SORBE \\ Department of Oncology, Faculty of Medicine and Health, Örebro University Hospital, Örebro SE-701 85, Sweden
}

Received December 28, 2014; Accepted December 4, 2015

DOI: $10.3892 / 01.2016 .4178$

\begin{abstract}
The present study investigated the predictive and prognostic impact of DNA ploidy together with other well-known prognostic factors in a series of non-endometrioid, high-risk endometrial carcinomas. From a complete consecutive series of 4,543 endometrial carcinomas of International Federation of Gynecology and Obstetrics (FIGO) stages I-IV, 94 serous carcinomas, 48 clear cell carcinomas and 231 carcinosarcomas were selected as a non-endometrioid, high-risk group for further studies regarding prognosis. The impact of DNA ploidy, as assessed by flow cytometry, was of particular focus. The age of the patients, FIGO stage, depth of myometrial infiltration and tumor expression of p53 were also included in the analyses (univariate and multivariate). In the complete series of cases, the recurrence rate was $37 \%$, and the 5-year overall survival rate was $39 \%$ with no difference between the three histological subtypes. The primary cure rate $(78 \%)$ was also similar for all tumor types studied. DNA ploidy was a significant predictive factor (on univariate analysis) for primary tumor cure rate, and a prognostic factor for survival rate (on univariate and multivariate analyses). The predictive and prognostic impact of DNA ploidy was higher in carcinosarcomas than in serous and clear cell carcinomas. In the majority of multivariate analyses, FIGO stage and depth of myometrial infiltration were the most important predictive (tumor recurrence) and prognostic (survival rate) factors. DNA ploidy status is a less important predictive and prognostic factor in non-endometrioid, high-risk endometrial carcinomas than in the common endometrioid carcinomas, in which FIGO and nuclear grade also are highly significant and important factors.
\end{abstract}

Correspondence to: Professor Bengt Sorbe, Department of Oncology, Faculty of Medicine and Health, Örebro University Hospital, Södra Grev Rosengatan, Örebro SE-701 85, Sweden E-mail: bengt.sorbe@ regionorebrolan.se

Key words: endometrial carcinoma, high-risk histology, serous carcinoma, clear cell carcinoma, carcinosarcoma, DNA ploidy, prognosis

\section{Introduction}

Endometrial carcinoma is the most common genital malignancy of women in developed countries. Globally, >280,000 new cases are diagnosed each year (1). The prognosis of endometrial carcinoma is generally thought to be favorable, predominantly due to detection and diagnosis in the early stages of disease. However, there are subtypes among endometrial carcinomas with poor prognosis and outcome after therapy (2).

Several types of tumors may be classified as high-risk. This includes serous adenocarcinoma, a highly malignanthistological type that was first described by Hendrickson et al (3) more than three decades ago. Clear cell carcinomas, similarly to renal carcinomas, also belong to a high-risk group and have a mutation profile more like that of serous carcinomas than common endometrioid carcinomas (4). During recent years, carcinosarcomas (mixed malignant mesodermal tumors) have been reclassified and moved from the uterine sarcoma group to the endometrial adenocarcinomas of high-risk type group (5). The incidence of recurrences, predominantly at distant sites $(65 \%)$, is more frequent for these high-risk types of non-endometrioid carcinomas than for endometrioid carcinomas $(6,7)$.

In certain studies, a number of early-stage serous carcinoma patients appear to have a good prognosis; however, reliable prognostic indicators are lacking (8). Investigation of predictive and prognostic factors (9) and definition of clinically relevant risk groups (10) are important steps to improve diagnosis, classification and treatment planning. To design clinical trials and to select optimal treatment, establishing reliable and reproducible risk groups is mandatory.

At present, no consensus exists regarding which predictive or prognostic factors should be used and how to combine them in the definition of various risk groups. Although numerous randomized phase III trials (11-16) have been presented in the literature during the last two decades, the results are difficult to compare and final conclusions remain uncertain and under debate. The low statistical power of certain studies dealing with predictive and prognostic factors has also prevented definitive conclusions regarding prognosis and optimal therapy. Analysis of data from large registry studies may allow circumvention of some of these problems $(9,10)$; however, scanty clinical data and selection bias may be shortcomings in these types of study.

Since 1980, a number of prospective randomized studies have been conducted to elucidate the value of external beam 
radiotherapy following surgery in early-stage endometrial carcinoma (11-15). The risk groups have differed between these trials, including low-risk, low to medium-risk, medium to high risk and high-risk groups. The type of primary surgery and staging have also varied, from no staging $(11,17)$ to staging with lymph node sampling or complete lymphadenectomy (pelvic and/or paraaortic) $(12,13)$. Subgroup analyses performed in these studies have suffered from low power (e.g., for pure high-risk cases), and no level-one data are generated. Vaginal brachytherapy, external beam pelvic radiation and adjuvant chemotherapy have been addressed in these studies $(15,16,18)$.

In the present retrospective study, a series of high-risk endometrial carcinomas was selected from a large consecutive, non-selected population of $>4,500$ endometrial carcinomas of International Federation of Gynecology and Obstetrics (FIGO) stages I-IV. Three types of high-risk tumors (serous carcinoma, clear cell carcinoma and carcinosarcoma) were selected among non-endometrioid carcinomas for further studies of predictive and prognostic factors, with special emphasis on the impact of DNA ploidy measured by flow cytometry.

\section{Patients and methods}

Patients. The Department of Gynecological Oncology, Örebro University Hospital recruited patients with all stages (FIGO I-IV) of endometrial carcinomas into this retrospective, observational study. The period of recruitment was between January 1975 and December 2009. In total, 4,543 patients were included. Postoperative external pelvic irradiation and/or vaginal brachytherapy were administered to the majority of the patients. For high-risk tumors, adjuvant platinum-based chemotherapy was also commonly administered.

From this consecutive series of endometrial carcinomas, tumors with high-risk pathology (serous carcinomas, clear cell carcinomas and carcinosarcomas) were selected for further analyses with regard to predictive and prognostic factors, with special focus on the importance of DNA ploidy. A total of 373 tumors fulfilled these high-risk criteria, of which 94 cases were serous carcinomas, 48 were clear cell carcinomas and 231 were carcinosarcomas. Further characteristics of the tumors are presented in Table I. Tumor size and lymphovascular space invasion were not included in the present study, as data regarding these variables were not regularly available in the pathology reports. Other types of high-risk tumors (endometrioid type) were not included in this review. All pathology reports were reviewed by one experienced pathologist from the Department of Pathology, Örebro University Hospital. The mean age of this subgroup of patients was 67.6 years (range, 32-94 years).

DNA ploidy status. DNA ploidy analysis was conducted by flow cytometry (FCM) as part of the routine histopathological evaluation of the endometrial carcinomas. In the majority of the cases, the S-phase fraction was not reported, and this variable was therefore not included in this study. The outcome was presented as diploid or non-diploid (including multiple aneuploid and tetraploid cases). A DNA diploid histogram was defined as containing a single major peak with a DNA index (DI) ranging from 0.90 to 1.10 ; one or more major peaks outside this range defined non-diploid histograms. A tetraploid histogram displayed a peak with a DI in the range of 1.80-2.20 ( $>15 \%$ of all cells measured). Among the endometrioid tumors, $21 \%$ were non-diploid (not further presented in this study), whilst serous carcinomas comprised $79 \%$ non-diploid cases, clear cell carcinomas comprised $50 \%$, and carcinosarcomas included $65 \%$ non-diploid cases. Tetraploid tumors were few, accounting for 47 of 1,601 evaluable cases (2.9\%). In clear cell carcinomas, no tetraploid cases were recorded.

Primary surgery. The primary surgery included a total abdominal hysterectomy, bilateral salpingo-oophorectomy, appendectomy, node sampling of enlarged lymph nodes and peritoneal washing with cytology. Lymphadenectomy was not routinely performedatthecentersreferring patients totheregional clinic. Surgeries were performed at the following institutions: Department of Gynecology and Obstetrics, Örebro University Hospital; Department of Gynecology and Obstetrics, Central Hospital, Karlstad; Department of Gynecology and Obstetrics, Eskilstuna; Department of Gynecology and Obstetrics, County Hospital, Karlskoga; Department of Gynecology and Obstetrics, County Hospital, Nyköping. All patients were subsequently referred to the gynecological oncology center for postoperative evaluation and treatment. The time interval between surgery and postoperative pelvic irradiation was 4-8 weeks. All patients were planned for a 10-year follow-up. The median follow-up period at the time of analysis was 64 months (range, 2-191 months) for surviving patients. During all visits, symptoms and signs associated with the therapy were recorded. However, the treatment-related side effects are not further presented in the current study. No patients were lost to follow-up.

Brachytherapy. For the brachytherapy treatments, microSelectron HDR machines (Elekta Instruments AB, Stockholm, Sweden) with an iridium source (Ir-192) were used. Plastic vaginal cylinders with a diameter of 20,25 or $30 \mathrm{~mm}$ were used as standard. The length of the vagina was measured from the vault to the level of introitus. The proximal two-thirds of the vaginal length were defined as the target volume. The dose per fraction was specified at a depth of $5 \mathrm{~mm}$ from the surface of the vaginal cylinder. Library dose plans that covered different vaginal lengths in steps of $10 \mathrm{~mm}$ and the different diameters of the cylinders were used. The dose calculations were made on the Nucletron Planning System (version 10; Elekta Instruments AB) and the PLATO Brachytherapy Planning System (version 14; Elekta Instruments AB). Six fractions were administered during an 8-day period. The dose per fraction was assigned as 2.5-3.0 Gy. Thus, the total doses delivered were 15.0-18.0 Gy. Recalculated to 2-Gy-equivalent doses, the total doses were 15.6-19.5 Gy at a depth of $5 \mathrm{~mm}$ $(\alpha / \beta=10.0 ; \alpha$, the linear term; $\beta$, the quadratic terms of the linear quadratic equation to describe the cell killing effect in radiotherapy).

External beam radiotherapy. External beam therapy was administered to patients with high-risk tumors. The target volume was the previous site of the uterus and adnexa, the parametrial tissues, the proximal two-thirds of the vagina, and the lymphatic drainage regions along the iliac vessels up to the promontory. The total median dose delivered to this volume 
was 46.0 Gy (range, 6.0-50.0 Gy) and daily fractions were 1.8-2.0 Gy.

Chemotherapy regimens. Following the completion of radiotherapy, platinum-based chemotherapy regimens [cisplatin $\left(50 \mathrm{mg} / \mathrm{m}^{2}\right)+$ doxorubicin $\left(50 \mathrm{mg} / \mathrm{m}^{2}\right)$; cisplatin $\left(75 \mathrm{~g} / \mathrm{m}^{2}\right)+$ cyclophosphamide $\left(750 \mathrm{mg} / \mathrm{m}^{2}\right)$; or carboplatin (AUC 5) + paclitaxel $\left.\left(175 \mathrm{mg} / \mathrm{m}^{2}\right)\right]$ were administered $(4$ cycles every 3 weeks for a duration of 9 weeks) to patients with high-risk tumors, including grade 3 endometrioid tumors with deep myometrial invasion.

Data management. All data were collected in a computerized database registry at the Regional Gynecological Oncology Center (Örebro, Sweden). The study was a retrospective, observational registry study. All analyzed data were retrieved from this clinical database and no further data were added from the patient records or from other sources.

Statistical analyses. For statistical analyses, survival curves were generated using the Kaplan-Meier method, and differences were tested with the log-rank test. The Pearson $\chi^{2}$ test was used for comparison of proportions, and an independent samples $t$-test or analysis of variance (ANOVA) for comparing the means of two or more groups. Multivariate analysis of prognostic factors was performed using the Cox proportional hazards model for survival data, and logistic regression analysis for binary outcome data (tumor recurrences). Best subset analysis was performed with a multivariate technique to find the most important prognostic factors and to find the most powerful and convenient combination of these factors. All P-values were based on two-sided tests, with $\mathrm{P}<0.05$ considered to indicate a statistically significant difference. The Statistica software package (StatSoft, Inc., Tulsa, OK, USA; version 12 ; 2013) was used for the statistical analyses.

\section{Results}

Patient series. The mean age of the complete series of patients was 67.6 years (range, 32-94 years). Patients with carcinosarcomas (mean age, 65.4 years) were significantly younger than patients with serous (mean age, 70.8 years) or clear cell (mean age, 71.7 years) carcinomas (ANOVA; $\mathrm{P}<0.001)$. Stage distribution demonstrated a significant difference, with more carcinosarcomas in stages I and IV [serous carcinomas 29/75 (38.7\%), clear cell carcinomas $18 / 38(47.4 \%)$ and carcinosarcomas 101/152 (66.5\%)], and more serous carcinomas and clear cell carcinomas in stages II and III [serous carcinomas 35/75 (46.7\%), clear cell carcinomas $15 / 38(39.5 \%)$ and carcinosarcomas $27 / 152$ $(17.8 \%)$ ] (Pearson $\left.\chi^{2} ; \mathrm{P}=0.004\right)$. In the complete series with available data on myometrial infiltration, significantly more serous carcinomas and clear cell carcinomas exhibited deep myometrial infiltration ( $\geq 50 \%$ of myometrial thickness) than carcinosarcomas (Pearson $\chi^{2} ; \mathrm{P}=0.049$ ). p53 positivity was detected in $94.7 \%$ of evaluable serous carcinomas, $60.0 \%$ of clear cell carcinomas, and $62.5 \%$ of carcinosarcomas; there was a statistically significant difference in the rate of p53-positive staining between the serous and clear cell carcinomas vs. the carcinosarcomas (Pearson $\chi^{2} ; \mathrm{P}=0.037$ ).
Table I. Characteristics of the series of high-risk tumors.

\begin{tabular}{|c|c|c|c|}
\hline Variable & $\mathrm{n}$ & $\%$ total & $\%$ evaluable \\
\hline \multicolumn{4}{|l|}{ Histology } \\
\hline Serous carcinoma & 94 & 25.2 & \\
\hline Clear cell carcinoma & 48 & 12.9 & \\
\hline Carcinosarcoma & 231 & 61.9 & \\
\hline \multicolumn{4}{|l|}{ Tumor stage (surgical) } \\
\hline I & 148 & 39.7 & 55.9 \\
\hline II & 30 & 8.0 & 11.3 \\
\hline III & 47 & 12.6 & 17.7 \\
\hline IV & 40 & 10.7 & 15.1 \\
\hline Unknown & 108 & 29.0 & \\
\hline \multicolumn{4}{|l|}{ Myometrial infiltration } \\
\hline$<50 \%$ & 83 & 22.3 & 54.3 \\
\hline$\geq 50 \%$ & 70 & 18.8 & 45.7 \\
\hline Unknown & 220 & 59.0 & \\
\hline \multicolumn{4}{|l|}{ DNA ploidy ${ }^{\mathrm{a}}$} \\
\hline Diploid & 34 & 9.1 & 32.1 \\
\hline Non-diploid & 72 & 19.3 & 67.9 \\
\hline Unknown & 267 & 71.6 & \\
\hline \multicolumn{4}{|l|}{ p53 status } \\
\hline Negative & 11 & 2.9 & 24.4 \\
\hline Positive & 34 & 9.1 & 75.6 \\
\hline Unknown & 328 & 87.9 & \\
\hline
\end{tabular}

${ }^{\mathrm{a} A s s e s s e d ~ b y ~ f l o w ~ c y t o m e t r y . ~}$

Endometrioid carcinomas, not further analyzed in this study, were p53-positive in $46.8 \%$.

Primary cure rate. In the complete series, primary cure (complete remission) was achieved in 292 out of 373 high-risk cases $(78.3 \%)$. The primary cure rates were $77.7 \%$ in serous carcinomas, $85.4 \%$ in clear cell carcinomas and $77.1 \%$ carcinosarcomas. Thus, there were no statistically significant differences between the three histopathological subtypes (Pearson $\chi^{2} ; \mathrm{P}=0.435$ ).

Recurrence rate. The overall recurrence rates of the complete series of high-risk endometrial carcinomas was 108 out of 373 cases $(29.0 \%)$, and 108 out of the 292 cases $(37.0 \%)$ that had achieved primary complete remission. Of these, 25 cases (6.7\%) had locoregional recurrence and 83 cases $(22.3 \%)$ had distant metastases. There were 8 single vaginal recurrences $(2.1 \%)$ and 17 pelvic recurrences outside the vagina (4.6\%). Among distant metastases, lung (46 cases; 12.3\%), abdomen (21 cases; $5.6 \%$ ), liver ( 5 cases; $1.3 \%$ ), bone ( 9 cases; $2.4 \%$ ), paraaortic nodes (3 cases; $0.8 \%$ ) and central nervous system ( 2 cases; $0.5 \%$ ) were the most frequent sites. Between the three histopathological high-risk types (serous carcinomas, clear cell carcinomas and carcinosarcomas), no significant differences were observed in the overall recurrence rate $(21.3 \%, 33.3 \%$ and $31.2 \%$, respectively; Pearson $\chi^{2} ; \mathrm{P}=0.158$ ) or in the sites of recurrences (Pearson $\chi^{2} ; \mathrm{P}=0.602$ ), with the exception of lung 
Table II. Logistic regression analyses. Predictive factors vs. overall recurrence rate.

\begin{tabular}{|c|c|c|c|}
\hline Predictive factor & OR & $95 \% \mathrm{CI}$ & P-value \\
\hline \multicolumn{4}{|l|}{ Univariate analyses } \\
\hline Age (per year) & 1.016 & $0.996-1.036$ & 0.129 \\
\hline FIGO stage (I-II vs. III-IV) & 0.567 & $0.251-0.884$ & 0.079 \\
\hline DNA ploidy ${ }^{a}$ & 0.562 & $0.092-1.032$ & 0.230 \\
\hline Infiltration $^{\mathrm{b}}$ & 0.384 & $0.024-0.743$ & 0.009 \\
\hline \multicolumn{4}{|l|}{ Multivariate analysis } \\
\hline Age (per year) & 1.024 & $0.960-1.089$ & 0.463 \\
\hline FIGO stage (I-II vs. III-IV) & 0.758 & $0.070-1.587$ & 0.744 \\
\hline DNA ploidy ${ }^{\mathrm{a}}$ & 0.576 & $0.060-1.212$ & 0.395 \\
\hline Infiltration $^{\mathrm{b}}$ & 0.355 & $0.316-1.027$ & 0.131 \\
\hline
\end{tabular}

${ }^{\mathrm{a}}$ Diploid vs. non-diploid; ${ }^{\mathrm{b}}<50 \%$ vs. $\geq 50 \%$ of myometrial thickness. $\mathrm{OR}$, odds ratio; $\mathrm{CI}$, confidence interval; FIGO, International Federation of Gynecology and Obstetrics.

Table III. Logistic regression analyses. Predictive factors vs. distant recurrence rate.

\begin{tabular}{|c|c|c|c|}
\hline Predictive factor & OR & $95 \% \mathrm{CI}$ & P-value \\
\hline \multicolumn{4}{|l|}{ Univariate analyses } \\
\hline Age (per year) & 1.008 & $0.987-1.029$ & 0.462 \\
\hline FIGO stage (I-II vs. III-IV) & 0.722 & $0.394-1.050$ & 0.330 \\
\hline DNA ploidy ${ }^{\mathrm{a}}$ & 0.630 & $0.143-1.117$ & 0.352 \\
\hline Infiltration ${ }^{\mathrm{b}}$ & 0.429 & $0.068-0.789$ & 0.021 \\
\hline \multicolumn{4}{|l|}{ Multivariate analysis } \\
\hline Age (per year) & 0.981 & $0.914-1.048$ & 0.573 \\
\hline FIGO stage (I-II vs. III-IV) & 1.006 & $0.255-1.757$ & 0.994 \\
\hline DNA ploidy ${ }^{\mathrm{a}}$ & 0.557 & $0.070-1.185$ & 0.361 \\
\hline Infiltration ${ }^{\mathrm{b}}$ & 0.302 & $0.360-0.963$ & 0.076 \\
\hline
\end{tabular}

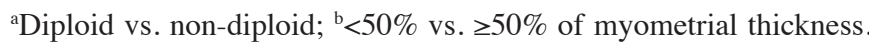
OR, odds ratio; CI, confidence interval; FIGO, International Federation of Gynecology and Obstetrics.

metastases; lung metastases were significantly more frequent $(19.1 \%)$ in carcinosarcomas than in serous $(5.5 \%)$ and clear cell carcinomas (12.2\%) (Pearson $\left.\chi^{2} ; \mathrm{P}=0.019\right)$. The median time to relapse was 24.8 months (range, 2-132 months). Out of 108 recurrences, $84(77.8 \%)$ were diagnosed within 3 years, and $97(89.8 \%)$ within 5 years. The mean age of patients with recurrences (68.1 years) was similar to the mean age of patients without recurrences (67.4 years).

Predictive factors for primary cure rate. DNA ploidy (diploid vs. non-diploid) was a statistically significant predictive factor for primary complete remission following therapy [logistic regression analysis; odds ratio, 2.90; 95\% confidence interval (CI), 2.36-3.43; $\mathrm{P}<0.050]$. The primary cure rates were $85.3 \%$ among diploid tumors and $66.7 \%$ among non-diploid tumors. Similar differences (diploid vs. non-diploid) in primary cure rate were observed for serous carcinomas ( 80 vs. 68\%), clear cell carcinomas (92 vs. $75 \%$ ) and carcinosarcomas (83 vs. $59 \%)$. FIGO stage was a strong and highly significant predictive factor for primary cure rate (Pearson $\chi^{2} ; \mathrm{P}<0.001$ ). The cure rate varied from $95 \%$ in stage I to $23 \%$ in stage IV. Depth of myometrial invasion was also a significant predictive factor for primary cure rate $(92 \%$ for superficial invasion and $77 \%$ for deep myometrial invasion; Pearson $\chi^{2} ; \mathrm{P}=0.013$ ). In a multivariate logistic regression analysis FIGO stage was the only significant $(\mathrm{P}=0.003)$ and independent predictive factor. Tumor expression of p53 ( $>30 \%$ staining of tumor cells) and age of the patients were not significant predictive factors for the primary cure rate of the tumor.

Predictive factors for tumor recurrences. In univariate and multivariate logistic regression analyses, tumor infiltration (superficial vs. deep) was a significant and independent predictive factor $(\mathrm{P}=0.016)$ in serous and clear cell carcinomas with regard to overall recurrence rate, following correction for age, tumor stage (FIGO) and DNA ploidy status. Age ( $\mathrm{P}=0.463)$, tumor stage $(\mathrm{P}=0.718)$ and DNA ploidy $(\mathrm{P}=0.895)$ were non-significant factors in this analysis as well as in univariate analyses. A best subset multivariate analysis confirmed that FIGO stage and depth of myometrial infiltration were the two most important predictive factors in a model with four included factors. Age and ploidy status had minor influence on the likelihood score of the model. When carcinosarcomas were included in the analysis, depth of tumor infiltration remained a significant predictive factor in univariate analysis $(\mathrm{P}=0.009)$ but not in multivariate analysis $(\mathrm{P}=0.131)$ (Table II). For distant recurrences, tumor infiltration was a significant $(\mathrm{P}=0.003)$ predictive factor in serous and clear cell carcinomas; however, this was not the case when carcinosarcomas were included in the analysis $(\mathrm{P}=0.076)$ (Table III). No significant predictive factors for distant recurrences and lung metastases could be identified in carcinosarcomas. DNA ploidy was a stronger predictive factor in carcinosarcomas than in clear cell carcinomas and serous carcinomas in univariate analyses with regard to overall recurrence rate. FIGO grade $(\mathrm{P}<0.001)$ and nuclear grade $(\mathrm{P}=0.005)$, highly significant and independent predictive factors in endometrioid carcinomas, were not applicable for the non-endometrioid carcinomas analyzed in the current study.

Survival analyses. The overall 5-year survival rate of the complete series of patients was 39.2\% (95\% CI, 33.8-44.6\%), whilst the cancer-specific survival rate was $46.9 \%$ (95\% CI, 41.1-52.7\%), and the recurrence-free survival rate was $37.2 \%$ (95\% CI, 31.8-42.6\%). No significant differences were identified between the three histopathological subtypes with regard to overall $\left(\chi^{2} ; \mathrm{P}=0.929\right)$ or cancer-specific $\left(\chi^{2} ; \mathrm{P}=0.873\right)$ survival rates (Fig. 1). The 5-year overall survival rate following any type and site of recurrence was only $3.3 \%$ in this series of high-risk carcinomas.

Prognostic factors for survival. Four prognostic factors (age, FIGO stage, tumor infiltration and DNA ploidy status) were analyzed with a Cox proportional hazard regression analysis (Table IV). In univariate analyses, all four factors were statistically significant with regard to overall, cancer-specific and recurrence-free survival rates. In multivariate analyses 
Table IV. Cox proportional hazard regression analyses. Prognostic factors vs. cancer-specific survival rate.

\begin{tabular}{lcccrr}
\hline Prognostic factor & $\beta$ & SE & HR & 95\% CI & P-value \\
\hline Univariate analyses & & & & & \\
$\quad$ Age (per year) & 0.034 & 0.007 & 1.035 & $1.021-1.049$ & $<0.001$ \\
FIGO stage (III-IV vs. I-II) & 0.620 & 0.084 & 3.456 & $2.483-4.811$ & $<0.001$ \\
DNA ploidy (non-diploid vs. diploid) & 0.441 & 0.152 & 2.414 & $1.331-4.379$ & 0.004 \\
Infiltration ( $\geq 50 \%$ vs. $<50 \%)^{\mathrm{a}}$ & 0.429 & 0.114 & 2.360 & $1.512-3.682$ & $<0.001$ \\
Multivariate analysis & & & & & \\
Age (per year) & 0.038 & 0.018 & 1.039 & $1.003-1.076$ & 0.035 \\
FIGO stage (III-IV vs. I-II) & 0.292 & 0.188 & 1.792 & $0.857-3.746$ & 0.121 \\
DNA ploidy (non-diploid vs. diploid) & 0.252 & 0.190 & 1.655 & $0.786-3.488$ & 0.185 \\
Infiltration ( $\geq 50 \%$ vs. $<50 \%)^{\mathrm{a}}$ & 0.098 & 0.198 & 1.217 & $0.560-2.644$ & 0.619 \\
\hline
\end{tabular}

${ }^{\text {a }}$ of myometrial thickness. $\beta$, parameter estimate; SE, standard error; HR, hazard ratio; CI, confidence interval; FIGO, International Federation of Gynecology and Obstetrics.

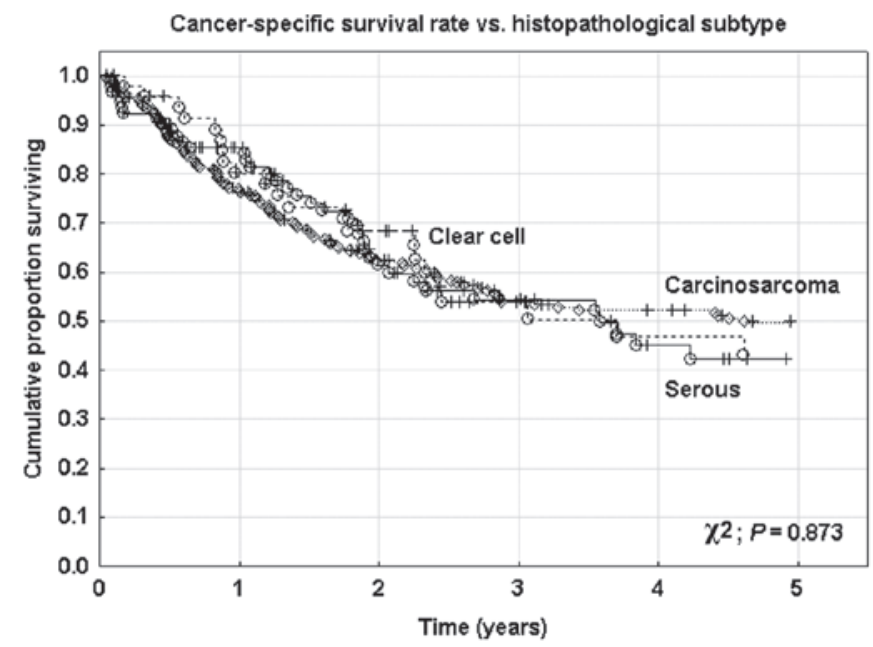

Figure 1. Type of histopathology had no significant impact on the cancer-specific survival rate in the complete series of high-risk endometrial carcinoma patients ( $\chi^{2}$ test; $\left.\mathrm{P}=0.873\right)$.

with all factors included, age alone was a significant and independent prognostic factor with regard to overall, cancer-specific and recurrence-free survival rates. DNA ploidy was a significant and independent factor when corrected for age, histopathological subtype and myometrial infiltration (Fig. 2), but not after correction for FIGO stage. DNA ploidy (non-diploid vs. diploid) had a stronger prognostic impact in carcinosarcomas (HR, 5.976) than in clear cell carcinomas (HR, 1.874) and serous carcinomas (HR, 1.456) with regard to cancer-specific survival rate. Tumor expression of p53 was a non-significant predictive and prognostic factor in all analyses.

\section{Discussion}

Non-endometrioid endometrial carcinomas have a significantly poorerprognosis than endometrioid carcinomas (2). Although the definition of high-risk carcinomas varies, the non-endometrioid histopathological subtypes (e.g., serous carcinoma, clear cell carcinoma and carcinosarcoma) are commonly included in this

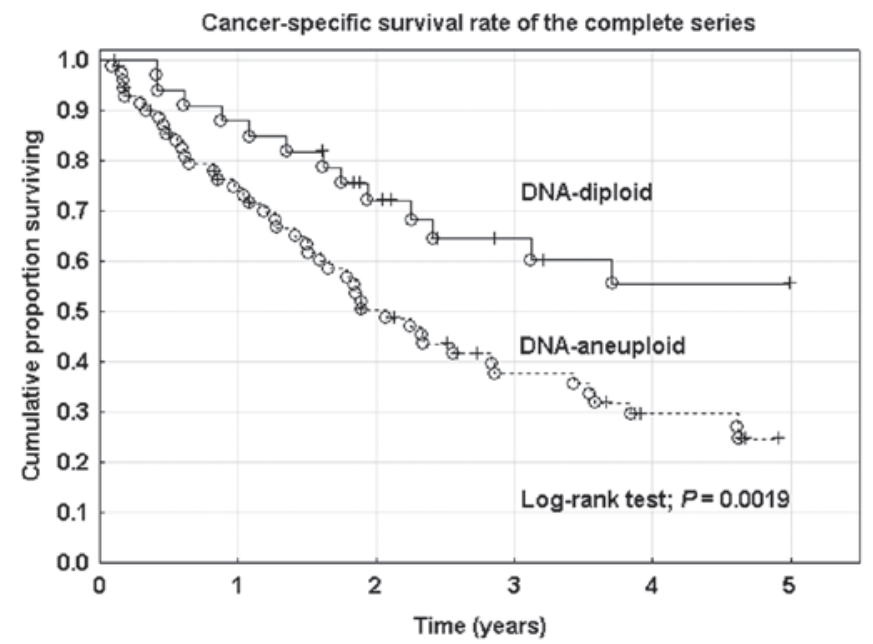

Figure 2. DNA-ploidy had a statistically significant impact on the cancer-specific survival rate in the complete series of high-risk endometrial carcinoma patients (log-rank test; $\mathrm{P}=0.0019)$.

risk group (19). Poorly differentiated endometrioid carcinomas with deep myometrial invasion also belong to the group of high-risk carcinomas of the endometrium. Identification of predictive and prognostic factors is an important prerequisite for definitions of risk groups in this disease and is the basis for design of clinical trials and for evaluation of the optimal treatment modalities for the individual patient (9). A number of predictive and prognostic factors in endometrioid carcinomas are described in the literature, certain of which are effective in the clinical definition of risk groups (10). However, for non-endometrioid carcinomas, the situation is different; predictive and prognostic factors are not so well-known and studied for these tumor types.

In the present study, 373 high-risk non-endometrioid tumors were selected from a large consecutive database of $>4,500$ cases of endometrial carcinomas. In all, 94 serous carcinomas, 48 clear cell carcinomas and 231 carcinosarcomas were identified. Predictive and prognostic factors, including the age of the patients, FIGO stage, histopathology, depth 
of myometrial infiltration, DNA ploidy and p53 expression, were analyzed with regard to primary cure rate of the tumor, recurrences rates (local, regional and distant) and survival rates (overall, cancer-specific and recurrence-free). Particular emphasis was placed on the prognostic impact of the DNA ploidy status measured by FCM.

In a prior study of endometrioid carcinomas, DNA ploidy was found to be a highly significant and independent risk factor together with the FIGO grade, histological subtype and depth of myometrial infiltration (9). For non-endometrioid carcinomas, the importance of these various predictive and prognostic factors is less clear (20), and this is also true for DNA ploidy status (21). A reason for this may be a significantly different distribution of the established predictive and prognostic factors between the endometrioid and the non-endometrioid tumors. Another reason may be the rarity of these tumors and, thus, the few published studies and small sample sizes of the individual histopathological subtypes. In the present study, three types of non-endometrioid carcinomas were included. A reason for this was the apparent similarities in the various outcome variables for these tumor types. Another reason is the morphological overlap amongst these groups, with problems often faced at diagnosis due to the observation of certain pathological features in all three tumor types (22). At present, carcinosarcomas, or mixed mesodermal tumors, are considered to be part of the high-risk endometrial carcinoma group rather than the endometrial sarcoma group (5). The high-risk group is a group of carcinomas with an extremely poor prognosis, few established prognostic factors, and without consensus regarding the optimal therapy. From our center and another Swedish center, we have presented data on a series of 322 endometrial carcinosarcomas and the clinical outcome following an adjuvant radiotherapy treatment schedule (23). In this series, 38\% recurrences occurred, with $28 \%$ at distant sites, and the overall survival rate was $30 \%$ (23). Combined radiotherapy and chemotherapy was the most efficient postoperative adjuvant therapy for locoregional tumor control (23).

The primary cure rates (complete remission) in the present series were similar for the three histopathological subtypes (77-85\%). DNA ploidy was a significant predictive factor for complete remission in the complete series and for the three subgroups. Tumor stage and depth of myometrial infiltration were also significant predictive factors. However, in a multivariate logistic regression analysis, FIGO stage was the only significant and independent factor.

The recurrence rate of the complete series was high, with $37 \%$ experiencing recurrence following primary complete remission, and the majority of the recurrences were of distant type. The overall recurrence rate was in the same range for the three tumor types; however, lung metastases were significantly more frequent (19\%) in carcinosarcomas. Uterine sarcomas are known to spread hematogenously, and the prognosis in advanced stages is extremely poor (23). In the current study, $78 \%$ of recurrences were diagnosed within the three years following therapy. DNA ploidy was a significant predictor for overall recurrence rate in carcinosarcomas, but not in serous and clear cell carcinomas. However, the most important predictive factor was myometrial infiltration, significant in univariate and multivariate analyses. Myometrial infiltration appeared to be a less important predictive factor in carcinosarcomas than in serous and clear cell carcinomas. The same result was seen for distant recurrences, where infiltration was significant in serous and clear cell carcinomas. For lung metastases, no significant risk factors could be identified except type of histopathology. FIGO grade and nuclear grade, known to be highly significant risk factors for recurrence and metastasis in endometrioid carcinomas, were not applicable in this group of non-endometrioid, high-risk carcinomas; according to the definitions, these tumors already have a high nuclear grade in the majority of the cases (3).

The survival of non-endometrioid carcinomas in this series was poor, with a 5-year overall survival rate of $39 \%$ in the complete series and with no significant differences between the histopathological subtypes. This survival rate is in agreement with a previous study (23). However, for early stage serous carcinomas, a favorable 5-year overall survival rate of $80 \%$ has been reported in the literature (8), which is not in agreement with the present results of 53\%.

Following tumor recurrence, survival was extremely poor, with a $3 \%$ overall 5 -year survival rate. The four prognostic factors studied (age, stage, myometrial infiltration and DNA ploidy) were all significant in univariate Cox proportional hazard regression analysis. DNA ploidy status was also significant and independent of age, histopathological subtype, and myometrial infiltration; however, it was not significant following correction for tumor stage. The two most important, significant and independent prognostic factors for cancer-specific survival rate were age and tumor stage. In a recent study from Norway on serous carcinomas, the only significant prognostic marker in univariate analyses was the $5 c$ excessive rate (ExR), a ploidy-related parameter used in the analysis of DNA-deviations, indicating an abnormal amount of DNA in tumor cells; it has also been demonstrated to be of prognostic importance in endometrial carcinoma and other tumor types. The explanation was that $5 \mathrm{c}$ ExR was high in proliferating tetraploid tumors and in aneuploid tumors with high DNA index (20). In an earlier study by Strang et al (24), $5 \mathrm{c}$ ExR was also a significant prognostic factor in endometrioid carcinomas. The 5c ExR DNA pattern has also been found to be a prognostic factor in other types of carcinomas (25). However, a different study revealed no association between DNA ploidy and survival in serous carcinomas (21). The advantages and disadvantages of FCM compared with image cytometry (20) have been discussed, and may be one explanation for different results regarding predictive and prognostic value of DNA ploidy in the presented studies. Image analysis seems to detect tetraploid and multiple aneuploid peaks more efficiently than FCM $(20,26,27)$. In contrast to the results of the current study, tumor stage and depth of infiltration did not predict prognosis in serous carcinomas in the Norwegian study (20), and a similar finding was reported by Goff et al (28) in a series of 50 serous carcinomas. Expression of estrogen and progesterone receptors, as well as of Ki-67 (a proliferation marker), were non-significant prognostic factors in the aforementioned study (28).

Tumor expression of $\mathrm{p} 53$, analyzed by immunohistochemistry, was not a significant predictive or prognostic factor in any of the analyses performed. Overexpression of p53 has been reported to be a prognostic factor in endometrioid carcinomas (29,30), but not in non-endometrioid disease, including serous carcinomas (31). 
For endometrioid carcinomas, it has been shown that DNA ploidy is an important predictive and prognostic factor and, if used in combination with the FIGO grade and type of histopathology, may replace myometrial invasion in the definition of preoperative high-risk cases requiring more extensive surgery (10). For high-risk non-endometrioid carcinomas, DNA ploidy is a significant predictive and prognostic factor on univariate analyses, but does not have the same importance and prognostic impact as for endometrioid carcinomas.

\section{Acknowledgements}

The author wishes to thank Mr. Peter Jansson, IT Coordinator at the Department of Oncology, Örebro University Hospital, for his work with the database and retrieval of the patient data included in this study. This work was supported by the research Foundation at the Department of Oncology, and the Foundation for Research in Gynecological Cancer, Örebro, Sweden.

\section{References}

1. Siegel R, Naishadham D and Jemal A: Cancer statistics, 2012. CA Cancer J Clin 62: 10-29, 2012.

2. Setiawan VW, Yang HP, Pike MC, McCann SE, Yu H, Xiang YB, Wolk A, Wentzensen N, Weiss NS, Webb PM, et al: Type I and II endometrial cancers: Have they different risk factors? J Clin Oncol 31: 2607-2618, 2013.

3. Hendrickson M, Ross J, Eifel P, Martinez A and Kempson R: Uterine papillary serous carcinoma: A highly malignant form of endometrial adenocarcinoma. Am J Surg Pathol 6: 93-108, 1982.

4. Hoang LN, McConechy MK, Meng B, McIntyre JB, Ewanowich C, Blake Gilks C, Huntsman DG, Köbel M and Lee $\mathrm{CH}$ : Targeted mutation analysis of endometrial clear cell carcinoma. Histopathology 66: 664-674, 2015.

5. McCluggage WG: Uterine carcinosarcomas (malignant mixed Mullerian tumors) are metaplastic carcinomas. Int J Gynecol Cancer 12: 687-690, 2002.

6. Sood BM, Jones J, Gupta S, Khabele D, Guha C, Runowicz C, Goldberg G, Fields A, Anderson P and Vikram B: Patterns of failure after the multimodality treatment of uterine papillary serous carcinoma. Int J Radiat Oncol Biol Phys 57: 208-216, 2003.

7. Pradhan M, Abeler VM, Danielsen HE, Sandstad B, Tropé CG, Kristensen GB and Risberg BÅ: Prognostic importance of DNA ploidy and DNA index in stage I and II endometrioid adenocarcinoma of the endometrium. Ann Oncol 23: 1178-1184, 2012.

8. Creasman WT, Odicino F, Maisonneuve P, Quinn MA, Beller U, Benedet JL, Heintz AP, Ngan HY and Pecorelli S: Carcinoma of the corpus uteri. FIGO 26th Annual Report on the Results of Treatment in Gynecological Cancer. Int J Gynaecol Obstet 95 (Suppl 1): S105-S143, 2006.

9. Kosary CL: FIGO stage, histology, histologic grade, age and race as prognostic factors in determining survival for cancers of the female gynecological system: An analysis of 1973-87 SEER cases of cancers of the endometrium, cervix, ovary, vulva and vagina. Semin Surg Oncol 10: 31-46, 1994.

10. Sorbe B: Predictive and prognostic factors in definition of risk groups in endometrial carcinoma. ISRN Obstet Gynecol 2012: 325790, 2012.

11. Creutzberg CL, Van Putten WL, Koper PC, Lybeert ML, Jobsen JJ, Wárlám-Rodenhuis CC, De Winter KA, Lutgens LC, van den Bergh AC, van de Steen-Banasik E, et al: Surgery and postoperative radiotherapy versus surgery alone for patients with stage-1 endometrial carcinoma: Multicentre randomised trial. PORTEC study group. Postoperative radiation therapy in endometrial carcinoma. Lancet 355: 1404-1411, 2000.

12. Keys HM, Roberts JA, Brunetto VL, Zaino RJ, Spirtos NM, Bloss JD, Pearlman A, Maiman MA and Bell JG; Gynecologic Oncology Group: A phase III trial of surgery with or without adjunctive external pelvic radiation therapy in intermediate risk endometrial adenocarcinoma: A gynecologic oncology group study. Gynecol Oncol 92: 744-751, 2004.
13. ASTEC/EN.5 Study Group, Blake P, Swart AM, Orton J, Kitchener H, Whelan T, Lukka H, Eisenhauer E, Bacon M, Tu D, et al: Adjuvant external beam radiotherapy in the treatment of endometrial cancer (MRC ASTEC and NCIC CTG EN. 5 randomised trials): Pooled trial results, systematic review and meta-analysis. Lancet 373: 137-146, 2009.

14. Nout RA, Smit VT, Putter H, Jürgenliemk-Schulz IM, Jobseb JJ, Lutgens LC, van der Steen-Banasik EM, Mens JW, Slot A, Kroese MC, et al: Vaginal brachytherapy versus pelvic external beam radiotherapy for patients with endometrial cancer of high-intermediate risk (PORTEC-2): An open-label, non-inferiority, randomised trial. Lancet 375: 816-823, 2010.

15. Sorbe B, Horvath G, Andersson H, Boman K, Lundgren C and Pettersson B: External pelvic and vaginal irradiation versus vaginal irradiation alone as postoperative therapy in medium-risk endometrial carcinoma-a prospective randomized study. Int J Radiat Oncol Biol Phys 82: 1249-1255, 2012.

16. Sorbe B, Nordström B, Mäenpää J, Kuhelj J, Kuhelj D, Okkan S, Delaloye JF and Frankendal B: Intravaginal brachytherapy in FIGO stage I low-risk endometrial cancer: A controlled randomized study. Int J Gynecol Cancer 19: 873-878, 2009.

17. Aalders J, Abeler V, Kolstad P and Onsrud M: Postoperative external irradiation and prognostic parameters in stage I endometrial carcinoma. Clinical and histopathologic study of 540 patients. Obstet Gynecol 56: 419-427, 1980.

18. Högberg T, Signorelli M, de Oliveira CF, et al: Sequential adjuvant chemotherapy and radiotherapy in endometrial cancer--results from two randomized studies. Eur J Cancer 36: 371-378, 2010.

19. Rutgers JK: Update on pathology, staging and molecular pathology of endometrial (uterine corpus) adenocarcinoma. Future Oncol 11: 3207-3218, 2015

20. Pradhan M, Davidson B, Abeler VM, Danielsen HE, Tropé CG, Kristensen GB and Risberg BÅ: DNA ploidy may be a prognostic marker in stage I and II serous adenocarcinoma of the endometrium. Virchows Arch 461: 291-298, 2012.

21. Kato DT, Ferry JA, Goodman A, Sullinger J, Scully RE, Goff BA, Fuller AF Jr and Rice LW: Uterine papillary serous carcinoma (UPSC): A clinicopathologic study of 30 cases. Gynecol Oncol 59: 384-389, 1995.

22. Garg K, Leitao MM Jr, Wynveen CA, Sica GL, Shia J, Shi W and Soslow RA: p53 overexpression in morphologically ambiguous endometrial carcinomas correlates with adverse clinical outcomes. Mod Pathol 23: 80-92, 2010.

23. Sorbe B, Paulsson G, Andersson S and Steineck G: A population-based series of uterine carcinosarcomas with long-term follow-up. Acta Oncol 52: 759-766, 2013.

24. Strang P, Stenkvist B, Bergström R, Stendahl U, Valdes del Campo $\mathrm{M}$ and Tribukait B: Flow cytometry and interactive image cytometry in endometrial carcinoma. A comparative and prognostic study. Anticancer Res 11: 783-788, 1991.

25. Grote HJ, Friedrichs N, Pomjanski N, Guhde HF, Reich O and Böcking A: Prognostic significance of DNA cytometry in carcinoma of the uterine cervix FIGO stage IB and II. Anal Cell Pathol 23: 97-105, 2001.

26. Danque PO, Chen HB, Patil J, Jagirdar J, Orsatti G and Paronetto F: Image analysis versus flow cytometry for DNA ploidy quantitation of solid tumors: A comparison of six methods of sample preparation. Mod Pathol 6: 270-275, 1993.

27. Huang Q, Yu C, Zhang X and Goyal RK: Comparison of DNA histograms by standard flow cytometry and image cytometry on sections in Barrett's adenocarcinoma. BMC Clin Pathol 8: 5, 2008.

28. Goff BA, Kato D, Schmidt RA, Ek M, Ferry JA, Muntz HG, Cain JM, Tamimi HK, Figge DC and Greer BE: Uterine papillary serous carcinoma: Patterns of metastatic spread. Gynecol Oncol 54: 264-268, 1994.

29. Salvesen HB, Iversen OE and Akslen LA: Prognostic significance of angiogenesis and $\mathrm{Ki}-67, \mathrm{p} 53$ and p21 expression: A population-based endometrial carcinoma study. J Clin Oncol 17: 1382-1390, 1999.

30. Alkushi A, Lim P, Quino-Parsons C and Gilks CB: Markers of proliferative activity are predictors of patient outcome for low-grade endometrioid adenocarcinoma but not papillary serous carcinoma of endometrium. Mod Pathol 15: 365-371, 2002.

31. Alkushi A, Kobel M, Kalloger SE and Gilks CB: High-grade endometrial carcinoma: Serous and grade 3 endometrioid carcinomas have different immunophenotypes and outcomes. Int J Gynecol Pathol 29: 343-350, 2010. 